\title{
Overview of Some Computational Techniques for Bioinformatics
}

This paper was downloaded from TechRxiv (https://www.techrxiv.org).

LICENSE

CC BY 4.0

SUBMISSION DATE / POSTED DATE

$14-10-2021 / 19-10-2021$

CITATION

Singh, Nehul; Verma, Karan (2021): Overview of Some Computational Techniques for Bioinformatics. TechRxiv. Preprint. https://doi.org/10.36227/techrxiv.16809817.v1

$\mathrm{DOI}$

10.36227/techrxiv.16809817.v1 


\section{Overview of Some Computational Techniques for Bioinformatics}

\author{
Nehul Singh \\ Student, \\ Department of Computer Science and Engineering, \\ IIIT Sricity, Chittoor, INDIA \\ nehul.s20@iiits.in
}

\author{
Karan Verma \\ Assistant Professor, \\ Department of Computer Science and Engineering, \\ NIT Delhi, INDIA \\ karanverma@nitdelhi.ac.in
}

\begin{abstract}
This article overviews importance of interdisciplinary technologies, which are facilitating tremendous growth for the bioinformatics applications. A summary in brief on development of bioinformatics areas and related technologies is presented. Emphasis mainly is given to discuss role of computer professionals and some computational tools to support the bioinformatics objectives. Challenges and issues faced by expected technological developments are also highlighted in view to adopt strategies for futuristic developments of bioinformatics.
\end{abstract}

Keywords - Interdisciplinary fields, bioinformatics, Computation tools and methods, Challenges and future trends

\section{INTRODUCTION}

Human genome sequencing [1] needs integrated and collective approach from biologists, mathematicians, genetic engineers, computer programmers and biochemist experts, etc. The growth in this field is taking the world towards an era, wherein all will be introduced to personal genome details to predict susceptible diseases and other health concerns [2]. However this will not be an easy task until unless all associated works in best planned and professional manner to achieve real-world applications of bioinformatics. Margaret $\mathrm{O}$. D., known as mother of bioinformatics, used combined applications of mathematics and computational methods for determination of protein sequence [3]. To achieve the targeted bioinformatics applications, clusters of scientist from various knowledge domains such as physiology, analytical chemistry, mathematics, genetic and computational engineering have been formed to work with common objectives. Thereafter similar practice have been in use to engage people from interdisciplinary fields of science and technologies for most of the bioinformatics task. Software professionals have been serving for medical science groups by developing bioinformatics tools to store, extract and to analyze useful information from biological database [4-6]. Such joint efforts resulted in development of tools for sequence search and analysis and models for structure predictions and molecular imaging/programs [7-9]. Most applications of bioinformatics contains processing of huge amount of data thereby a suitable operating system is needed to provide faster processing of the data and to enable users to work with large computing resources for simultaneous tasks. Fortunately, developments in the areas of computer science have created variety of such platforms with unique and favorable features to support bioinformatics applications [10-13]. With this article, we intend to assess the current challenges and opportunities in the bioinformatics market. The article overviews basic concepts and important features of Bioinformatics and describes associationship of computational tools with it. In last, paragraphs are written to discuss popular bioinformatics tools followed by limitations and future aspect of the field.

\section{CONTRIBUTION OF COMPUTER SCIENCE FOR BIOINFORMATICS}

The computation tools can be useful for development of scientific methods to understand the biological data, which eventually leads to applications of bioinformatics. Computation professionals are supporting bioinformatics by performing activities for biological data base with use of algorithmic tools to facilitate analytical characterization. Perhaps they are actively participating for development of bioinformatics infrastructures to manage the biological information. Figure 1 depicts major related fields of bioinformatics, whereby computer professionals have been engaged to render their services. Human Genome project [14] is a recent example, whereby computational tools are helping such methods to interpret and to record billions of basic pairs of the human DNA system by analysing huge amount of genetic data. Statistical analytical methods are needed to interpret the biological data, which can be developed jointly by people associated with interdisciplinary science and technical fields. However to achieve best use of the bioinformatics, software developers and biologists must be able to exchange and understand each other opinion on requisite knowledge areas such as statistics, logic and programming, genomics, genetics, and molecular biology. Therefore a computer engineer may contribute to bioinformatics by applying his skills to develop better and 
speedy methods to enhance understanding of genetic, molecular and clinical information for biologists.

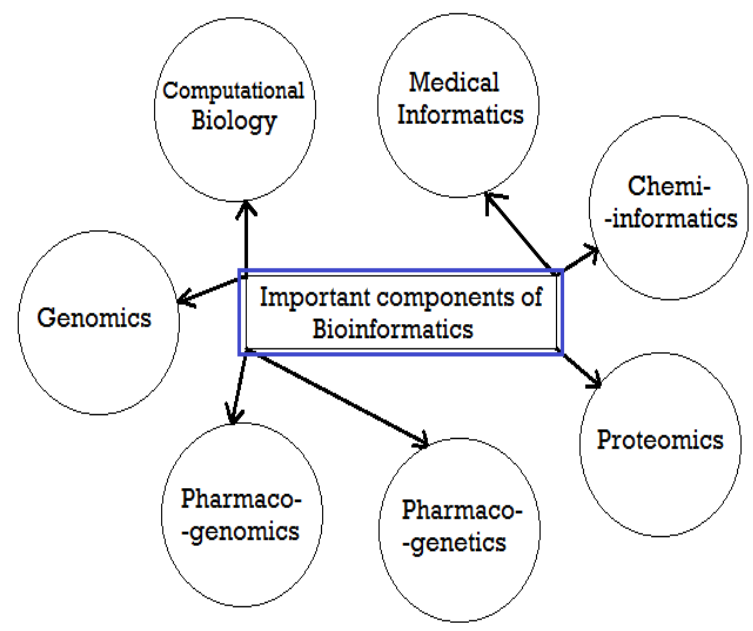

Fig. 1 Different related fields of Bioinformatics

In past one decade, there have been tremendous growths in engagement of computing professionals to analyze and manage a large amount of data generated by the health science service sectors. Figure 2 depicts some of the roles; a computer science professional can perform to support healthcare services eventually leading in line with objectives of the bioinformatics. Same can be stated as summarized below [1516];

- To develop soft-tools for visualization of data generated from the research on the genetic composition of humans.

- To correlate the cells activities with novel medicines to establish their uses treatments for various diseases.

- To design algorithms and to develop analytical soft methods to analyze and storage the biological data such as data of genes, proteins, drug ingredients and metabolic pathways.

- To perform simulation for constructed database and to analyze the sequence, functions, and structures.

- To extend help in executing research and development tasks for discovery of novel biological events with use of computational techniques and methods.

Software professionals have been utilizing their soft skills to develop computer-based biological simulation technologies and solutions. Various programming languages; Java, Perl, Python, etc. have been emerged as key players to support various bioinformatics activities. Bioinformatics projects such as PatternHunter, BioJava, BioPerl, Biopython, BioXML, BioCorba, etc. are some recent examples [17]. This field is so fascinating, that many renowned academic institutions and research computing organisation have now started degree courses covering interdisciplinary skills of computer science and biomedical technologies [18-20]. Graduates and researchers with such interdisciplinary skills are futuristic employee for various areas of medical science such as pharmaceutical research centers, clinical care, development of biomedicine and special drugs, clinical diagnosis, preventive medicine, etc. Perhaps recent developments in field of big data management, large data analytics, artificial intelligence, etc. are more suitable for their applications with biomedical areas [4] [21].

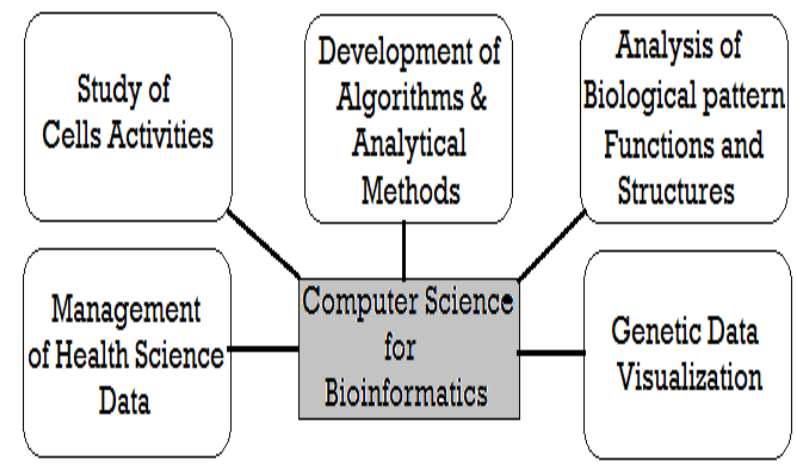

Fig. 2 Pivotal roles of Computer Science for Bioinformatics

In other words, bioinformatics needs professional who are familiar with healthcare environment and capable to utilize their computation skills to work with biomedical data. On the other hand, a health science professional can also be trained with computational skills to support the bioinformatics applications

\section{SUITABLE PLATFORMS AND COMPUTATIONAL TOOLS}

To perform computation using any program or method, we need a supportive operating system (OS), which host the tool and helps in managing the central processing unit, storage devices and components and printers by establishing a user interface and to execute and provide services. In most bioinformatics applications, we need to perform several tasks simultaneously; a supporting OS coordinates all of this to make sure each program gets what it needs [22]. Computer professionals develop soft-tools to extract information from biological databases and to perform sequence or structural analysis apart from discovery of processing methods for novel drugs and treatment analysis [15]. Most of such soft-tools have been made available as open-source products for distribution to the wider scientific and health community. Reviews on some suitable operating platforms and bioinformatics tools are discussed in brief in subsections as follows;

\section{Operating Systems:}

Modern operating systems use a graphical user interface, which enable users to access all resources of computing device using a combination of graphics and text. Most of the bioinformatics applications involve dealing with large database maintained by clusters of machines, therefore a professional need to apply skills to access the data from 
remote location. Account information such as name, password, and hostname for each user enable requisite remote access by a suitable OS such as Linux, Mac and MS Windows. Computational professions have preferred Linux platform on other operating systems to perform most of the tasks of bioinformatics due to its features like open-source, stability, less crash rate, suitable for multithreading operations and time-sharing systems, etc. [22]. Some bioinformatics tools work easily with Windows OS, perhaps .NET and $\mathrm{C}++$ programmers find more comfortably to work with it. The speed to process the biological data is an important aspect, whereby Linux has an edge over the Windows. On the other hand, to analyze and interpret the data; Windows has been preferred due to its suitability with common programming languages such as Python, Ruby and Perl. Support to highquality typesetting system (e.g. LaTex) and scripting languages (e.g. perl, python) and features like illustrator and compatibility with Linux makes Mac OS another option to work with bioinformatics applications. Working with Mac OS is also recommended, if we need to accommodate tools and problems for limited user-level interaction.

\section{Bioinformatics soft-tools:}

Various types of software tools have been in practice to perform tasks related to bioinformatics and to support technical aspects of medical sciences. Lots of programming methods have been proposed to support the objectives of Genome analysis. These tools enable medical practitioners to identify the group of genes and their interactions in order to predict the possible mutations eventually identification of possible disorders and diseases. In recent years, bioinformatics tools have been used for genome sequencing to study the arrangement of DNA, RNA, and proteins in the Covid-19 virus to understand its changing nature [23-24]. In last 2 decades, computations professional have proposed a number of soft-tools which are supporting objectives of the bioinformatics applications. Some of popular soft-tools are as follows;

- BLAST [25]: Basic local alignment search tool for searching of sequence using heuristic algorithms.

- GALAXY [26]: Data integration and analytical processes. Application in Gene expression, proteomics, etc.

- Ascalaph Designer [27]: Molecular modeling and simulation. Useful for study of lipid bilayers, ionic liquids, proteins and nucleic acids.

- EMBOSS [28]: European molecular biology open software suite for molecular biology. Useful for sequence alignment, database search, protein motif identification, domain analysis, etc.

- Geworkbench [29]: Biological software for genomic data analysis. Useful for visualization of Gene expression data, sequence and structuring.

- FastQC [30]: Useful for quality checks of sequencing pipeline raw data.
Programmers have developed tools using various programming languages, which are suitable to perform molecular dynamics such as BioPerl, BioJava, Biopython, BioPHP, BioRuby, etc. [31]. Many other soft-tools have been in use for numerous applications of bioinformatics such as MetaQUAST, MetaVelvet, MetAMOS, MetaWatt, PHYSCIMM, CONCOCT etc. [32].

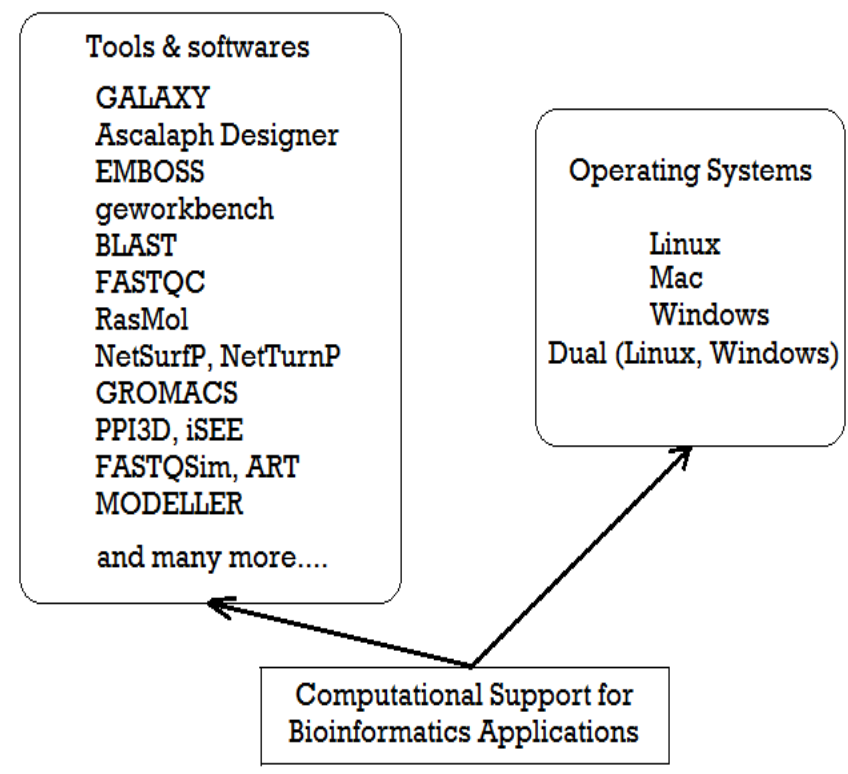

Fig. 3 Summary of useful operating systems and soft-tolls for bioinformatics

Some tools have been used for protein sequences predominantly; for example, NetSurfP [33] is suitable for surface accessibility and secondary structure predictions, while the NetTurnP [34] is useful to predict the Beta-turn regions in protein sequences. Few more popular biological soft-tools are summarized below;

- RNA structure prediction programs: mfold/UNAFold, Vienna RNA Websuite, NUPACK, Pfold, CONTRAfold, etc. [35]

- Predicting Protein-DNA Interactions: DISPLAR, BindN+, DISIS, DNABindR, etc. [36]

- Molecular imaging/modeling programs: Mage, RasMol, Chime, etc. [37]

- Molecular dynamics simulation: GROMACS, AMBER, OpenMM, NAMD, CHARMM etc. [38]

- Protein-protein interactions: PPI3D, DisruPPI, MutaBind, iSEE, mCSM-PPI2 [39]

- Multi-template homology modeling: ModSeg/ENCAD, SWISS-MODEL, NEST, MODELLER [40]

- Simulation of genomic next-generation sequencing data: CuReSim, FASTQSim, ART, simNGS, EAGLE, etc. [41].

From last two years, we have been going through uncontrollable outbreak of severe acute respiratory syndrome coronavirus (SARS-COV-2). Since then, computational 
professionals are placing joint efforts with healthcare scientist to design and develop methods and bioinformatics tools to study, detect and to suggest possible line of treatment for ongoing Pandemic COVID-19. Their efforts have resulted in several detection, annotation and tracking tools such as PriSeT, CoVPipe, VADR, Pfam, Rfam COVID-19, CoVGLUE, Covidex, etc. and in drug development processes such as VirHostNet, CORDITE, CoVex, etc. [23].

\section{CHALLENGES AND FUTURISTIC APPROACHES}

Since the objectives of the bioinformatics are going to serve enhancement of medical services for mankind, therefore utmost care is most important to come up with new treatment guidelines in view of bioinformatics generated information. Process modeling to predict transcription initiation and termination in a Genome, RNA /alternative splicing and to predict cellular response to external stimuli, etc. precisely will be common challenges for bioinformatics professionals. Accurate characterization of protein-DNA/RNA, proteinprotein recognition codes and ab-initio structures will enable professional to suggest correct health line procedures. Growth of any new technology depends upon the availability of workforce to work on, it is therefore expected that a huge investment of money and thoughts shall be made to develop adequate infrastructures and educational education setups. Perhaps, need of the hour is to introduce fundamentals of bioinformatics at the level of school and undergraduate education so as to nurture young minds to be the futuristic bioinformatics professionals.

The field of bioinformatics shall be going to open vast opportunities for interested professionals to work on evolutionary model building, data storage/retrieval, database structures, annotation and phylogenic analysis. Professionals having exposures with artificial intelligence and machine learning tools shall also be able to contribute for data mining, analyses of complex systems, genetic circuits, etc. Software programmers will also be useful to perform metabolic computing, and to process with information content in DNA, RNA, protein sequences and structures. It is evident from various sources such as leading newspapers, medical magazines, technical reports, etc that the bioinformatics is undergoing an enormous expansion and creating lots of service, research and development opportunities for interested professionals. In last two decades, there has been a substantial growth in the number of professionals shifting their interest to work with bioinformatics applications all across the globe. Apart from data mining and evaluation of prediction models, computer professional will see more opportunity to associate with professional clusters working for novel drug and vaccine developments, pharmacology, immune informatics, centralized bio-data analysis, etc. Recent technological advancements in computer science engineering such as big data management, artificial intelligence, machine learning, quantum computing, etc. have also been proving as a catalyst for generation of more opportunities for bioinformatics related activities.

\section{CONCLUSION}

We have reviewed fundamentals and latest happening in the field of bioinformatics covering the suitable operating platforms and popularly used soft-tools to achieve its objectives. A detailed discussion on relevant common challenges in developing the bioinformatics-based applications and possible growth in future. In times to come, the Bioinformatics will be indispensable for bio-data analysis and discovery of novel drugs, vaccines and treatment methods. It is evident from regular and genuine reports that the global market for bioinformatics shall be achieving around $10 \%$ compound annual growth rate for the period of 2020-2025. This shall be achieved by collaborative approach at the global level with a tremendous growth in relevant academic and research areas. Regular reports and technical articles on new advanced technologies and novel innovations in bioinformatics solutions with possible investments and funding opportunities will make this field more fascinating among the professionals. Sharing of information on impact analysis of Pandemic like situations such as Covid-19 on bioinformatics companies and adopted management strategies to continue with discovery of novel biological methods, drug discovery and development, etc. will certainly help for small technological business incubation centres and start-up companies in relevant fields.

\section{ACKNOWLEDGMENT}

We are thankful to all authors and contributors, whose work highlighting the bioinformatics tools and applications have enlightened us with knowledge to prepare this article. We also acknowledge the Pandemic Covid-19 Era, subsequent lockdown and virtual study mode to motivate us for this study.

\section{References}

[1] Chial, H. DNA sequencing technologies key to the Human Genome Project. Nature Education 1(1):219, (2008).

[2] Hood, L., Rowen, L. The Human Genome Project: big science transforms biology and medicine. Genome Med 5, 79 (2013). https://doi.org/10.1186/gm483.

[3] W.J.S. Diniz, F. Canduri, 2017. Bioinformatics: an overview and its applications. Genet. Mol. Res. 16 (1): gmr16019645, http://dx.doi.org/10.4238/gmr16019645.

[4] Dash, S., Shakyawar, S.K., Sharma, M. et al. Big data in healthcare: management, analysis and future prospects. J Big Data 6, 54 (2019). https://doi.org/10.1186/s40537-019-0217-0

[5] Ivan Merelli, Horacio Perez-Sanchez, Sandra Gesing, Daniele D'Agostino "Managing analysis and integrating Big data in medical bioinformatics: Open problems and Future Perspectives", BioMed Research International, vol. 2014, Pages 13, (2014). https://doi.org/10.1155/2014/134023.

[6] Ison, J., Ienasescu, H., Chmura, P. et al. The bio.tools registry of software tools and data resources for the life sciences. Genome Biol 20 , 164 (2019). https://doi.org/10.1186/s13059-019-1772-6

[7] Kuhlman, B., Bradley, P. Advances in protein structure prediction and design. Nat Rev Mol Cell Biol 20, 681-697 (2019). https://doi.org/10.1038/s41580-019-0163-x. 
[8] Jianyi YangIvan Anishchenko, Hahnbeom Park, Zhenling Peng, Sergey Ovchinnikov and David Baker "Improved protein structure prediction using predicted interresidue orientations" PNAS, 117 (3): 1496-1503, January 21, 2020, https://doi.org/10.1073/pnas.1914677117.

[9] Vallat B, Madrid-Aliste C, Fiser A (2015) Modularity of Protein Folds as a Tool for Template-Free Modeling of Structures. PLoS Comput Biol 11(8): e1004419. https://doi.org/10.1371/journal.pcbi.1004419.

[10] Suryakant Chaubal 'Operating System Concepts” Lulu publisher, Pages 146, ISBN-13:978-1411688599, April 2006

[11] Stecher G, Tamura K, Kumar S. "Molecular Evolutionary Genetics Analysis (MEGA) for macOS" Mol Biol Evol. 37(4):1237-1239, April 2020, https://doi.org/10.1093/molbev/msz312.

[12] Morais D, Roesch LFW, Redmile-Gordon M, Santos FG, Baldrian P, Andreote FD, Pylro VS. "BTW-Bioinformatics Through Windows: an easy-to-install package to analyze marker gene data" PeerJ. 6:e5299. July 2018, https://doi.org/10.7717/peerj.5299.

[13] Magda Mielczarek, Bartosz Czech, Jarosław Stańczyk, Joanna Szyda, Bernt Guldbrandtsen "Extraordinary Command Line: Basic Data Editing Tools for Biologists Dealing with Sequence Data" Letter, Open Bioinformatics Journals, 13: 137-145, 2020, https://doi.org/10.2174/1875036202013010137.

[14] Hood, L., Rowen, L. The Human Genome Project: big science transforms biology and medicine. Genome Med 5, 79 (2013). https://doi.org/10.1186/gm483.

[15] Jacques Cohen "Bioinformatics - An introduction for computer scientists" ACM Computing Surveys, 36(2):122-158, June 2004, https://doi.org/10.1145/1031120.1031122.

[16] Mario Cannataroa, Rodrigo Weber dos Santosb, Joakim Sundnesc "Biomedical and Bioinformatics Challenges to Computer Science: Bioinformatics, Modeling of Biomedical Systems and Clinical Applications" ICCS 2011, Procedia Computer Science 4: 1058-1061, 2011, https://doi.org/10.1016/j.procs.2011.04.112.

[17] Ryu, Tae-Wan "Benchmarking of BioPerl, Perl, BioJava, Java, BioPython, and Python for Primitive Bioinformatics Tasks and Choosing a Suitable Language" International Journal of Contents, 5(2): 6-15, June 2009, https://doi.org/10.5392/IJOC.2009.5.2.006.

[18] Muhammad Muddassir Ali, Muhammad Hamid, Muhammad Saleem, Saadia Malik, Natash Ali Mian, Muhammad Ahmed Ihsan, Nadia Tabassum, Khalid Mehmood, Furqan Awan, "Status of Bioinformatics Education in South Asia: Past and Present". BioMed Reseach International, Vol. 2021, Article ID: 5568262, Pages: 9, 2021. https://doi.org/10.1155/2021/5568262.

[19] David D Lent, Korenna M Estes, Alexandria K Hansen, Increasing Faculty Involvement in the Undergraduate Interdisciplinary Learning Experience, Integrative and Comparative Biology, 2021; icab109, https://doi.org/10.1093/icb/icab109.

[20] Guillermo Molina Recio, Laura García-Hernández, Rafael Molina Luque, Lorenzo Salas-Morera "The role of interdisciplinary research team in the impact of health apps in health and computer science publications: a systematic review" BioMed Eng OnLine 15, 77 (2016). https://doi.org/10.1186/s12938-016-0185-y.

[21] Bohr, Adam, and Kaveh Memarzadeh. "The rise of artificial intelligence in healthcare applications." Artificial Intelligence in Healthcare (2020): 25-60. https://doi.org/10.1016/B978-0-12-818438-7.00002-2.

[22] Jurate daugelaite, Aisling O Driscoll, Roy D. Sleator "An overview of multiple sequence alignments and cloud computing in Bioinformatics" International Scholarly Research Notices, Vol. 2013, Article ID: 615630, Pages 14, 2013, https://doi.org/10.1155/2013/615630.

[23] Franziska Hufsky, Kevin Lamkiewicz, Alexandre Almeida, Abdel Aouacheria, Cecilia Arighi, Alex Bateman, et al. "Computational strategies to combat COVID-19: useful tools to accelerate SARS-CoV-2 and coronavirus research" Briefings in Bioinformatics, 22(2): 642-663, March 2021, https://doi.org/10.1093/bib/bbaa232.

[24] Isabel, S., Graña-Miraglia, L., Gutierrez, J.M., Cedoljub BundalovicTorma, Helen E. Groves, Marc R. Isabel, et al. Evolutionary and structural analyses of SARS-CoV-2 D614G spike protein mutation now documented worldwide. Sci Rep 10, $14031 \quad$ (2020). https://doi.org/10.1038/s41598-020-70827-z
[25] Feagan, L., Rohrer, J., Garrett, A., Heather Amthauer, Ed Komp, David Johnson, et al. "Bioinformatics process management: information flow via a computational journal" Source Code Biol Med 2, 9 (2007). https://doi.org/10.1186/1751-0473-2-9.

[26] Vahid Jalili, Enis Afgan, Qiang Gu, Dave Clements, Daniel Blankenberg, Jeremy Goecks, James Taylor, Anton Nekrutenko, The Galaxy platform for accessible, reproducible and collaborative biomedical analyses: 2020 update, Nucleic Acids Research, 48 (W1): W395-W402, 02 July 2020, https://doi.org/10.1093/nar/gkaa434.

[27] Nida Tabassum Khan. "Clinical Bioinformatics: Linking Pharmacogenomics with Informatics". Acta Scientific Pharmaceutica Sciences 2.3 (2018): 67-70.

[28] Anna-Lena Lamprecht, Stefan Naujokat, Tiziana Margaria, Bernhard Steffen "Semantics-based composition of EMBOSS services" J Biomed Semant 2, S5 (2011). https://doi.org/10.1186/2041-1480-2-S1-S5.

[29] Aris Floratos, Kenneth Smith, Zhou Ji, John Watkinson, Andrea Califano "geWorkbench: an open source platform for integrative genomics" Bioinformatics, 26 (14): 1779-1780, 1779-1780, 2010 https://doi.org/10.1093/bioinformatics/btq282.

[30] Leggett RM, Ramirez-Gonzalez RH, Clavijo BJ, Waite D, Davey RP. "Sequencing quality assessment tools to enable data-driven informatics for high throughput genomics", Front Genet. 2013; 4:288. 2013, https://doi.org/10.3389/fgene.2013.00288

[31] Milicchio, F., Rose, R., Bian, J., Min, J., \& Prosperi, M. "Visual programming for next-generation sequencing data analytics." BioData mining, vol. 9:16, 27 Apr. 2016, https://doi.org/10.1186/s13040-0160095-3.

[32] Despoina D. Roumpeka, R. John Wallace, Frank Escalettes, Ian Fotheringham, Mick Watson "A Review of Bioinformatics Tools for Bio-Prospecting from Metagenomic Sequence Data" Front. Genet., 06 March 2017, https://doi.org/10.3389/fgene.2017.00023.

[33] Petersen B, Petersen TN, Andersen P, Nielsen M, Lundegaard C. "A generic method for assignment of reliability scores applied to solvent accessibility predictions" BMC structural biology, vol. 9:51, $31 \mathrm{Jul}$. 2009, https://doi.org/10.1186/1472-6807-9-51.

[34] Petersen B, Lundegaard C, Petersen TN. "NetTurnP--neural network prediction of beta-turns by use of evolutionary information and predicted protein sequence features" PloS one vol. 5:11 e15079. 30 Nov. 2010, https://doi.org/10.1371/journal.pone.0015079.

[35] Schroeder SJ "Advances in RNA structure prediction from sequence: new tools for generating hypotheses about viral RNA structure-function relationships" Journal of Virology, 83(13): 6326-6334, July 2009, https://doi.org/10.1128/jvi.00251-09.

[36] Milanowska, K., Rother, K., \& Bujnicki, J. M. "Databases and bioinformatics tools for the study of DNA repair" Molecular biology international, 475718, 2011, https://doi.org/10.4061/2011/475718.

[37] Michelle A. Harris, Ronald F. Peck, Shannon Colton, Jennifer Morris, Elias Chaibub Neto, Julie Kallio "A Combination of Hand-held Models and Computer Imaging Programs Helps Students Answer Oral Questions about Molecular Structure and Function: A Controlled Investigation of Student Learning" CBE-Life Sciences Education, 8 (1): 29-43, ISSN (online): 1931-7913, October 2017 https://doi.org/10.1187/cbe.08-07-0039.

[38] Scott A. Hollingsworth, Ron O.Dror "Molecular Dynamics Simulation for All” Neuron, 99 (6): 1129-1143, 19 September 2018 , https://doi.org/10.1016/j.neuron.2018.08.011.

[39] Carlos Eduardo Sequeiros-Borja, Bartłomiej Surpeta, Jan Brezovsky "Recent advances in user-friendly computational tools to engineer protein function" Briefings in Bioinformatics, vol. 22, issue 3, May 2021, https://doi.org/10.1093/bib/bbaa150.

[40] Meier A, Soding J "Automatic Prediction of Protein 3D Structures by Probabilistic Multi-template Homology Modeling” PLoS Comput Bio 11(10): e1004343, 2015, https://doi.org/10.1371/journal.pcbi.1004343.

[41] Escalona, M., Rocha, S. \& Posada, D. "A comparison of tools for the simulation of genomic next-generation sequencing data" Nat Rev Genet 17, 459-469 (2016). https://doi.org/10.1038/nrg.2016.57. 